\title{
The Low-lying Quadrupole Collective Excitations of Ru and Pd Isotopes*
}

\author{
K. Zając, L. Próchniak and K. Pomorski \\ Institute of Physics, The Maria Curie-Sktodowska University, \\ pl. M. Curie-Sktodowskiej 1, 20-031 Lublin, Poland \\ S.G. Rohoziński and J. Srebrny \\ Department of Physics, Warsaw University, \\ Hoża 69, 00-681 Warsaw, Poland
}

June 14, 2021

\begin{abstract}
Quadrupole excitations of even-even $\mathrm{Ru}$ and $\mathrm{Pd}$ isotopes are described within microscopic approach based on the general collective Bohr model which includes the effect of coupling with the pairing vibrations. The excitation energies and E2 transition probabilities observed in ${ }^{104-114} \mathrm{Ru}$ and ${ }^{106-110} \mathrm{Pd}$ are reproduced in the frame of the calculation containing no free parameters. Particularly interesting are ${ }^{104} \mathrm{Ru}$ and ${ }^{106-110} \mathrm{Pd}$ where good agreement with very rich information based on Coulomb excitation experiments is achieved.
\end{abstract}

PACS 21.60.Ev, 23.20.-g, 27.60.+j

${ }^{*}$ The work is supported in part by KBN contract No. 2 P03B 06813. 


\section{Introduction}

The microscopic approach to the general collective Bohr hamiltonian [1, 2] was formulated many years ago in order to investigate the coupling between nuclear rotations and surface oscillations. This microscopic Bohr hamiltonian still offers a consistent way to interpret nuclear collective modes directly referring to single-particle degrees of freedom. Within this approach one can construct realistic models of the collective quadrupole excitations in different nuclei without introducing free parameters and fitting procedures what is rather inevitable if calculations are made in the frame of phenomenological descriptions (as, for instance, in [3] or [4]). This important feature allows to investigate the structure of collective bands in the wide range of even-even nuclei using no adjustable parameters except those fixed for all nuclei: the single-particle potential parameters and the strength of the residual pairing interaction.

However, the microscopic approach to nuclear collective excitations has not been as useful as it could be because it failes in reproducing the experimental level densities. As it is known since a long time [2], the energies of excited levels obtained within microscopic Bohr model are in general larger than the measured ones. The possible reason for such a disagreement found in Ref. [5] was the absence of an important collective degree of freedom connected with the pairing vibrations [6]. The strong influence of pairing correlations on collective nuclear movements contributes to the coupling between quadrupole and pairing vibrations. For that reason the pairing energy gap $\Delta$ should not be artifficially fixed at its equilibrium point (found from the BCS equations) when studying nuclear collective excitations. In fact, $\Delta$ should be treated as a collective variable representing changes in the pairing field due to coherent nucleon movements composing collective modes.

Recently we have developed in Ref. [0] an approximation based on the general Bohr hamiltonian which includes the average effect of pairing dynamics to the quadrupole excitations. Within this method (described in Section 2) we have obtained the energies of excited levels close to their experimental positions for the chains of neutron-deficient $\mathrm{Te}, \mathrm{Xe}, \mathrm{Ba}, \mathrm{Ce}$ and $\mathrm{Nd}$ isotopes. In the present work we would like to show the results of calculations performed in the region of neutron-rich deformed nuclei from the mass region of $A \approx 100$. In Section 3 we discuss the spectroscopic structure of $A=104-114$ $\mathrm{Ru}$ isotopes which caused recently some controversions about the role of the nonaxial $\gamma$ deformation. We have studied also collective properties of the $A=106-110 \mathrm{Pd}$ isotopes

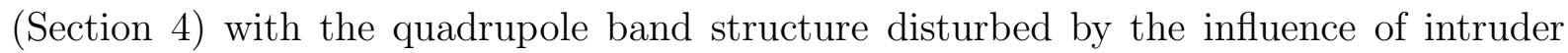
states. For both groups of nuclei we have obtained a significant succes in reproducing the experimantal data. The results presented in this work confirm the importance of the coupling with the pairing vibrations in proper treatment of the nuclear collective quadrupole excitations. 


\section{The model}

In order to take into account the exact coupling between quadrupole shape oscillations and pairing collective modes we should solve the full "quadrupole + pairing" problem, what is rather troublesome because of 9 degrees of freedom to deal with: two intrinsic variables $\beta$ and $\gamma$ parametrizing the shape of nuclear surface, three Euler angles for its orientation in space (denoted in short as $\Omega$ ), two gap parameters $\Delta^{p}$ and $\Delta^{n}$ for protons and neutrons and two corresponding gauge angles $\phi^{p}, \phi^{n}$. Assuming that the coupling between quadrupole and pairing variables is weak enough to neglect mixing terms we can derive an approximate solution. The approximate collective hamiltonian consists of two known terms (the operator $\hat{\mathcal{H}}_{\text {int }}$ mixing quadrupole and pairing variables will be neglected in further calculations):

$$
\hat{\mathcal{H}}_{\mathrm{CQP}}=\hat{\mathcal{H}}_{\mathrm{CQ}}\left(\beta, \gamma, \Omega ; \Delta^{p}, \Delta^{n}\right)+\hat{\mathcal{H}}_{\mathrm{CP}}\left(\Delta^{p}, \Delta^{n} ; \beta, \gamma\right)+\hat{\mathcal{H}}_{\text {int }} .
$$

Here and in all following formulas the variables placed after a semi-colon should be treated as parameters (they do not appear in differential operators). The first term $\hat{\mathcal{H}}_{\mathrm{CQ}}$ describes quadrupole oscillations and rotations of a nucleus and it takes the form of the generalized Bohr hamiltonian [1, 2, 7] (in Ref. [7] it was denoted as $\hat{\mathcal{H}}_{\text {coll }}$ ):

$$
\hat{\mathcal{H}}_{\mathrm{CQ}}=\hat{\mathcal{T}}_{\text {vib }}\left(\beta, \gamma ; \Delta^{p}, \Delta^{n}\right)+\hat{\mathcal{T}}_{\text {rot }}\left(\beta, \gamma, \Omega ; \Delta^{p}, \Delta^{n}\right)+V_{\text {coll }}\left(\beta, \gamma ; \Delta^{p}, \Delta^{n}\right)
$$

where $V_{\text {coll }}$ is the collective potential. The kinetic vibrational energy reads

$$
\begin{gathered}
\hat{\mathcal{T}}_{\text {vib }}=-\frac{\hbar^{2}}{2 \sqrt{w r}}\left\{\frac{1}{\beta^{4}}\left[\partial_{\beta}\left(\beta^{4} \sqrt{\frac{r}{w}} B_{\gamma \gamma} \partial_{\beta}\right)-\partial_{\beta}\left(\beta^{3} \sqrt{\frac{r}{w}} B_{\beta \gamma} \partial_{\gamma}\right)\right]+\right. \\
\left.+\frac{1}{\beta \sin 3 \gamma}\left[-\partial_{\gamma}\left(\sqrt{\frac{r}{w}} \sin 3 \gamma B_{\beta \gamma} \partial_{\beta}\right)+\frac{1}{\beta} \partial_{\gamma}\left(\sqrt{\frac{r}{w}} \sin 3 \gamma B_{\beta \beta}\right) \partial_{\gamma}\right]\right\}
\end{gathered}
$$

and the rotational energy is

$$
\hat{\mathcal{T}}_{\text {rot }}=\frac{1}{2} \sum_{k=1}^{3} \hat{I}_{k}^{2} / \mathcal{J}_{k} .
$$

The intrinsic components of the total angular momentum are denoted as $\hat{I}_{k},(k=1,2,3)$, while $w$ and $r$ are the determinants of the vibrational and rotational mass tensors. The mass parameters (or vibrational inertial functions) $B_{\beta \beta}, B_{\beta \gamma}$ and $B_{\gamma \gamma}$ together with moments of inertia $\mathcal{J}_{k},(k=1,2,3)$ depend, in general, on intrinsic variables $\beta, \gamma$ and pairing gap values $\Delta^{p}, \Delta^{n}$. All inertial functions are determined from a microscopic theory. We

apply the standard cranking method to evaluate the inertial functions assuming that the nucleus is a system of nucleons moving in the deformed mean field (Nilsson potential) which interact through monopole pairing forces. According to the known formulas (given for example in [7]) the inertial functions can be expressed in terms of matrix elements of derivatives of the nuclear hamiltonian on collective variables, single-particle energies (depending on deformation point $\beta, \gamma$ ) and occupation probabilities of the single-particle 
levels obtained by solving the BCS equations for given gap values $\Delta^{p}, \Delta^{n}$. The collective potential is calculated within Strutinsky macroscopic-microscopic method [8].

It should be stressed that in the minimum of the BCS energy with respect of $\Delta$ one obtains the usual equilibrium gap values $\Delta_{e q}^{p}, \Delta_{e q}^{n}$ and the operator

$$
\hat{\mathcal{H}}_{\mathrm{CQ}}\left(\beta, \gamma, \Omega ; \Delta^{p}=\Delta_{e q}^{p}, \Delta^{n}=\Delta_{e q}^{n}\right)
$$

becomes exactly the same as the Bohr hamitonian known from Ref. [1] or [2].

For a given nucleus the second term in Eq. (四) describes collective pairing vibrations of systems of $Z$ protons and $A-Z$ neutrons

$$
\hat{\mathcal{H}}_{\mathrm{CP}}=\hat{\mathcal{H}}_{\mathrm{CP}}^{Z}+\hat{\mathcal{H}}_{\mathrm{CP}}^{A-Z}
$$

and it can be expressed in the following form [6, 9]:

$$
\hat{\mathcal{H}}_{\mathrm{CP}}^{\mathcal{N}}=-\frac{\hbar^{2}}{2 \sqrt{g(\Delta)}} \frac{\partial}{\partial \Delta} \frac{\sqrt{g(\Delta)}}{B_{\Delta \Delta}(\Delta)} \frac{\partial}{\partial \Delta}+V_{\text {pair }}(\Delta),
$$

where $\mathcal{N}=Z, \Delta=\Delta^{p}$ for protons and, respectively, $\mathcal{N}=A-Z, \Delta=\Delta^{n}$ for neutrons. The functions appearing in the hamiltonian (7), namely the pairing mass parameter $B_{\Delta \Delta}(\Delta)$, the determinant of the metric tensor $g(\Delta)$ and the collective pairing potential $V_{\text {pair }}(\Delta)$ are determined microscopically at each deformation point $\beta, \gamma$ according to the formulas given in Ref. 99. The approximate projection of the BCS wave function on correct particle number [7, 10] is applied within the calculation.

Solving the eigenproblem of the collective pairing hamiltonian (7) one can find the pairing vibrational ground-state wave function $\Psi_{0}^{\mathcal{N}}$ and the ground-energy $E_{0}^{\mathcal{N}}$ at each deformation point. The most probable value of the energy gap $\Delta_{v i b}$ corresponds to the maximum of the probability of finding a given gap value in the collective pairing groundstate (namely the maximum of the function $g(\Delta)\left|\Psi_{0}^{\mathcal{N}}(\Delta)\right|^{2}$ ). As it is shown in Fig. 1 the $\Delta_{v i b}$ is shifted towards smaller gaps from the equilibrium point $\Delta_{e q}$ determined by the minimum of $V_{\text {pair }}$ (or by the BCS formalism). Such a behaviour of the pairing ground state function $\Psi_{0}^{\mathcal{N}}$ is due to the rapid increase of pairing mass parameter $B_{\Delta \Delta}$ and it appears in most cases. In general the ratio of $\Delta_{v i b}$ to $\Delta_{e q}$ is of about 0.7 .

However, the exact diagonalization of the collective hamiltonian (1) still remains difficult because of the dimension of the needed basis. But as far as only low-lying nuclear excitatons are taken into account one can consider the coupling between quadrupole and pairing collective degrees of freedom diagonalizing the hamiltonian (2) just in the point corresponding to the most probable gaps $\Delta_{v i b}^{p}, \Delta_{v i b}^{n}$, i.e.

$$
\hat{\mathcal{H}}_{\mathrm{CQP}} \approx \hat{\mathcal{H}}_{\mathrm{Q}}\left(\beta, \gamma, \Omega ; \Delta^{p}=\Delta_{v i b}^{p}, \Delta^{n}=\Delta_{v i b}^{n}\right)
$$

It means that all collective functions appearing in Eq. (8) are calculated using the most probable pairing gap values for protons and for neutrons instead the equilibrium ones as 
it used to be when the expression (5) had been diagonalized. The collective potential also depends on the most probable pairing gaps and, in addition, it is slightly corrected by the pairing vibrational ground-state energies $E_{0}^{Z}$ and $E_{0}^{A-Z}$ coming from the collective pairing term (7). In order to solve the eigenproblem of the hamiltonian $\hat{\mathcal{H}}_{\mathrm{CQP}}$ we calculate its matrix elements using the functions $\tilde{\Phi}_{j}^{I M}(\beta, \gamma, \Omega)$ where $I$ means the angular momentum, $M=-I, \ldots, I$ while $j$ means the set of additional quantum numbers. The basis $\tilde{\Phi}_{j}^{I M}(\beta, \gamma, \Omega)$ was obtained [7] following the approach of Libert and Quentin [1].

Other collective properties are also characterized by matrix elements of appropriate operators evaluated in the basis mentioned above. All collective operators (as the electric quadrupole moments) are determined microscopically using cranking formulas and the most probable pairing gaps $\Delta_{v i b}^{p}, \Delta_{v i b}^{n}[7]$.

The approximation described above is rather crude but, as we would like to exemplify in next sections, it includes the main effect (at least on average) of the coupling with the pairing vibrational mode. This procedure improves significantly the accuracy in reproducing the experimental data but it introduces no additional parameters into the model. Our calculations were done using the standard Nilsson single particle potential with the shell dependent parametrization found in Ref. [12]. For the pairing strength we applied the same estimates as used in Ref. [7]: $G=g_{0} \mathcal{N}^{2 / 3}$, where $\mathcal{N}=Z$ or $\mathcal{N}=A-Z$ for protons or neutrons respectively and $g_{0}=0.26 \hbar \omega_{0}$.

\section{$3 \quad \mathrm{Ru}$ isotopes}

Recent investigations of the collective properties of even-even neutron-rich $\mathrm{Ru}$ isotopes done in Ref. [13] and Ref. [14] caused a discussion about the role of the $\gamma$ deformation in this region. These nuclei appear to be generally triaxial: both, predicted 15 and experimentally deduced by fitting to the rigid triaxial rotor model [13] the equilibrium $\gamma$ values are close to $20^{\circ}$. On the other hand, there were made some observations [14] suggesting that $\mathrm{Ru}$ isotopes are rather $\gamma$-soft with prolate equilibrium shapes. In consequence different (even opposite) phenomenological approaches as the rigid triaxial rotor model (asymmetric rotor model) [4, 13, 16], the rotation-vibration model [4, 14, 16] and more general (but still based on the geometrical approach) collective model adopted in Ref. [4] were applied in order to interpret collective bands in $\mathrm{Ru}$ isotopes, each with a comparable success. It seems that the reason of such a situation may be connected with some essential difficulties in determining the equilibrium shapes. The collective potentials calculated within the model described in Section 2 appear generally triaxial (see Fig. 2), what is in agreement with the expectations [13, 15. However, the bottoms of the potential energy surfaces are very flat what can lead to the considerable uncertainty in location of the minima. Anyway, a $\gamma$-softness of the nuclei in question seems to be beyond all doubts.

Diagonalizing the hamiltonian (8) with such collective potentials we have obtained the 
excitation energies and the corresponding wave functions of ${ }^{104-114} \mathrm{Ru}$ (dependent on $\beta$ and, rather smoothly, on $\gamma$ deformation). The calculated energies of positive parity states of ${ }^{104} \mathrm{Ru}$ presented in Fig. 3 (see the part marked as "new") agree very well with the measured ones. Small discrepancies appearing for the higher $0^{+}$states may be connected with the absence of mixing terms in the model hamiltonian (11) or with the restrictions imposed on the basis (see Section 2). For the $8_{2}^{+}$level the difference between theoretical and experimental [17, 18] energy is rather due to the observed mixture of two-particle mode. For comparison we present also (see the part of Fig. 3 marked as "old") the excitation energies obtained with the original Bohr hamiltonian (expression 5 in Section 2). As one can learn from Fig. 3 the improvement in reproducing the experimental data caused by coupling with the pairing vibrations is really significant.

To get a real proof of the proper identification of obtained wave functions we have investigated their electromagnetic properties. The experimental evidence gathered by Coulomb excitations in Ref. 17, 18 for ${ }^{104} \mathrm{Ru}$ is fairly rich so we can verify our results in details. Calculated diagonal matrix elements of the quadrupole electric operator in the excited states of this nucleus are compared with the observed ones in Fig. 4. Theoretical values follow the experimental data, the agreement is good enough to reproduce almost exactly the the changement of the quadrupole moment of ${ }^{104} \mathrm{Ru}$ along the bands.

In Fig. 5 we present theoretical and experimental reduced E2 transition probabilities. The transitional probabilities observed in the ground-state and $\gamma$ bands were measured by J. Stachel et al. [17] while E2 transitions involving the $0_{2}^{+}$band were investigated by J. Srebrny et al. [18]. The results of calculations show that the wave functions obtained in our approximation really represent low-lying excited states of ${ }^{104} \mathrm{Ru}$. We have obtained too small values of the probabilities of E2 transitions between members of the ground-state band but they are still close to the data. Besides, for the $\gamma$ and $0_{2}^{+}$bands we have reached a very good agreement with the observed E2 transition probabilities. Even some weak probabilities of the band-to-band transitions obtained in this work are nicely confirmed by the experiment.

We have obtained a similar agreement with the experimental data [13, 14, 19, 20] for excited states in ${ }^{106,108} \mathrm{Ru}$ (Fig. 6) and ${ }^{110,112} \mathrm{Ru}$ (Fig. 7) isotopes. There are some discrepancies, for example the calculated $6_{1}^{+}$level descends a little below the experimental point disturbing the smooth experimental dependence of the energy on the angular momentum. It looks like a sudden change in the structure of the ground-state band energies which is not seen in the experiment. Perhaps it is due to the lack of components of higer multipolarities in our model. Another inaccuracy appears in ${ }^{112} \mathrm{Ru}$, where the moment of inertia in the $\gamma$ band is too large with respect to observed one. In spite of those relatively small discrepancies we have got a realistic description of the collective band structure in all considered $\mathrm{Ru}$ isotopes, even for such a very neutron reach nucleus as ${ }^{114} \mathrm{Ru}$ (Fig. 8).

As was mentioned before, the lowering of quadrupole transitions between the neigh- 
bouring members of a ground-state band seems to be a general feature of our model in the region of neutron-deficient nuclei [7]. This tendency occurs also in ${ }^{106-114} \mathrm{Ru}$ isotopes: few available experimental data points are situated (see Fig. 9) above the theoretical points. The experimental evidence of the reduced transition probabilities in heavy $\mathrm{Ru}$ isotopes is rather poor but we suppose that our predictions should be as realistic as for

${ }^{104} \mathrm{Ru}$, especially the results obtained for $\gamma$ bands. We did not find any data concerning quadrupole moments in the excited ${ }^{106-114} \mathrm{Ru}$ states but we would like to present in Fig. 10 the predicted (and rather characteristic for all considered $\mathrm{Ru}$ isotopes) dependence of the diagonal E2 diagonal matrix elements on the angular momentum.

In the last years there were made rather intensive theoretical investigations of ${ }^{108-112} \mathrm{Ru}$ within different phenomenological models, e.g. the triaxial rotor model [13, 4], the rotation -vibration model [14, 4] and the generalized collective model (GCM) [4]. All those models have numerous parameters fitted to the data (for instance 8 parameters for each nucleus in the GCM). Thus the direct comparison of earlier results with ours which are obtained without adjustable parameters does not seem to be very useful. Nevertheless, within approach presented here the calculated energies of excited levels in ${ }^{108-112} \mathrm{Ru}$ are situated almost as close to their experimental positions as in Ref. [4].

The accuracy in reproducing the observed band structure suggests almost pure quadrupole nature of low-lying states in the $Z=44, A=104-112$ nuclei. The further suggestion is that their shapes are in general triaxial but soft or very $\gamma$-soft. The coupling between quadrupole and pairing collective degrees of freedom plays an essential role in description of the neutron-rich $\mathrm{Ru}$ isotopes but, on the other hand, the shape and pairing vibrations can be treated separately to some extent.

\section{$4 \quad \mathrm{Pd}$ isotopes}

As $\mathrm{Ru}$ isotopes were treated as triaxial rotors, the $\mathrm{Pd}$ isotopes used to be interpreted in terms of vibrational modes. But the careful Coulomb excitation experimental investigations (e.g. 21, 22, 23]) have changed this picture and showed that $\mathrm{Pd}$ isotopes exhibit a rather complicated structure. The IBA-2 model [3, 22] has been applied in order to interpret the band-like spectroscopic structure and to calculate quadrupole electric transitions (see Fig. 13). But it should be mentioned that the IBA-2 model made use of 15 (and even more) free parameters while the simpler IBA-1 version was unable to describe the Pd nuclei because of a competition between $\mathrm{SU}(5)$ and $\mathrm{O}(6)$ boson limits [22, 25].

Some complications in the band structure of $\mathrm{Pd}$ isotopes arise because of the intruder states [22, 24] and their interaction with "normal" single-particle states. But according to the experimental suggestions the transitional $Z=46$ nuclei should be interpreted using collective potentials soft in both $\beta$ and $\gamma$ deformations. The collective Bohr hamiltonian (see Section 2) automatically takes into account the rotation-vibration coupling and it 
seems to be an appropriate approach. Of course, also here the Bohr hamiltonian should be generalized in order to describe the influence of pairing vibrations, at least on average.

The ${ }^{106-110} \mathrm{Pd}$ have been described in the frame of exactly the same model which was referred in Section 2 and applied to $\mathrm{Ru}$ isotopes in Section 3. As a result we have obtained the low-lying positive parity states, their energies, quadrupole moments and transitions for $\mathrm{Pd}$ isotopes. Collective potentials obtained for ${ }^{106} \mathrm{Pd}$ and ${ }^{108} \mathrm{Pd}$ are shown in Fig. 11. The potential surfaces are very shallow and it is almost imposible to determine exactly $\beta$ and $\gamma$ equilibrium values. The uncertainty of nuclear equilibrium shapes as well as the structure of wave functions corresponding to low excitation energies testify the softness of $\mathrm{Pd}$ isotopes in both, $\beta$ and $\gamma$ deformations.

The theoretical energy levels are compared to the four quasi-rotational bands observed in ${ }^{106} \mathrm{Pd},{ }^{108} \mathrm{Pd}$ (Fig. 12) and ${ }^{110} \mathrm{Pd}$ (Fig. 13). It is visible that ground state bands and $\gamma$ bands with their characteristic staggering are reproduced very well. For remaining bands the agreement is not so good. But the $0_{2}^{+}$bands observed in ${ }^{108} \mathrm{Pd}$ and ${ }^{110} \mathrm{Pd}$ have different features than it is expected for collective states. According to Ref. [22, 24] the large deformations estimated experimentally for these bands can be due to the particlehole configurations connected with the presence of intruder states. On the other hand, there are strong experimental indications [22] that the nature $0_{2}^{+}$band in ${ }^{106} \mathrm{Pd}$ and the system of levels built on the $0_{3}^{+}$state in ${ }^{110} \mathrm{Pd}$ are very similar. Following this suggestion we decided to compare the theoretical $0_{2}^{+}, 2_{3}^{+}, 4_{3}^{+}$and $6_{3}^{+}$levels to the members of the experimentally found $0_{3}^{+}$band. Such a new theoretical $0_{3}^{+}$band matches the observed one as is pictured in Fig. 13.

The rearrangement could be justified by investigations of the electromagnetic properties of considered states. The reduced E2 transition probabilities between members of redefined theoretical $0_{3}^{+}$band agree much better with the experimental data (see Fig. 14) than probabilities calculated in the ground state band (which tend to be too small as in the other nuclei [7]). However, the observed diagonal matrix elements of E2 operator in all states represented in Fig. 15 are reproduced quite well within our approximation.

Quadrupole electric transitions and diagonal matrix E2 elements for ${ }^{106,108} \mathrm{Pd}$ are compared to the experimental data in Fig. 16-18. The agreement reached in this work is not worse than in the IBA-2 calculations using fitting procedures [3, 22, 25]. In fact, we could find that our results obtained with no free parameters are even more reliable.

The structure of $\mathrm{Pd}$ isotopes is more complicated than the $\mathrm{Ru}$ ones: several different degrees of freedom are expected in the low-lying positive parity states in the $\mathrm{Pd}$ nuclei including non-collective modes as the proton two- particle- two- hole excitations. Nevertheless, we were able to interpret nuclear states hardly described within geometrical pictures of the vibrator or asymmetric rotor or within IBA-1 model [25]. Our results support the picture of the Pd isotopes as $\beta$ - and $\gamma$-soft quadrupole deformed nuclei easily adopting triaxial shapes. We found that the coupling betweeen quadrupole and pairing 
vibrations in $\mathrm{Pd}$ isotopes appears very important and plays the similar role as in $\mathrm{Ru}$ nuclei.

\section{$5 \quad$ Summary and Conclusions}

The general collective Bohr hamiltonian including the average effect of coupling with the pairing vibrations was applied to neutron-rich $\mathrm{Ru}$ and $\mathrm{Pd}$ isotopes. The collective properties of low-lying excited states were interpreted within model containing no adjustable parameters. The resulting excitation energies agree well with the measured values and, moreover, a resonable agreement with the spectroscopic data was reached in description of their electromagnetic properties. We should mention that our approximation works even better for $A \approx 100$ neutron rich nuclei than in the region of transitional neutron-deficient $\mathrm{Te}, \mathrm{Xe}$ or Ba isotopes [7].

Some discrepancies found in comparison with the experimental data are partly due to the non-collective nature of some excited states. Disagreements were observed especially for the configurations involving intruder orbitals (the $0_{2}^{+}, 0_{3}^{+}$bands in ${ }^{108,110} \mathrm{Pd}$ ). Some discrepancies could be also connected with the restrictive assumptions made when the model hamiltonian was formulated (see Section 2). The coupling between quadrupole and pairing vibrations was approximated by shifting of the pairing gap to its most probable value. This simplified way may be too drastic to get appropriate values of reduced E2 transition probabilities. Especially too low values of the calculated transition probabilities between members of ground state band are supposed to be connected with the approximate treatment of the coupling between pairing and quadrupole vibrations.

Nevertheless, the results of our calculations are close to the experimental data. This agreement confirm the importance of the collective pairing mode in the theoretical description of even-even nuclei. We expect that the model could be improved by more careful treatment of terms mixing the intrinsic variables $\beta$ and $\gamma$ with pairing gaps in the collective hamiltonian.

\section{References}

[1] M. Baranger and K. Kumar, Nucl. Phys. A122 (1968) 241.

[2] S.G. Rohoziński, J. Dobaczewski, B. Nerlo-Pomorska, K. Pomorski and J. Srebrny, Nucl. Phys. A292 (1977) 66.

[3] P. Van Isacker, G. Puddu, Nucl. Phys. A348 (1980) 125.

[4] D. Troltenier, J.P. Draayer, B.R.S. Babu, J.H. Hamilton, A.V. Ramayya, V.E. Oberacker, Nucl. Phys. A601 (1996) 56. 
[5] S. Piłat and K. Pomorski, Nucl. Phys. A554 (1993) 413.

[6] D.R. Bès, R.A. Broglia, R.P.J. Perazzo and K. Kumar, Nucl. Phys. A143 (1970) 1.

[7] L. Próchniak, K. Zając, K. Pomorski, S.G Rohoziński, J. Srebrny, Nucl. Phys. A in print.

[8] S.G. Nilsson, C.F. Tsang, A. Sobiczewski, Z. Szymański, S. Wycech, C. Gustaffson, I.L. Lamm, P. Möller and B. Nilsson, Nucl. Phys. A131 (1969) 1.

[9] A. Góźdź, K. Pomorski, M. Brack and E. Werner, Nucl. Phys. A442 (1985) 50.

[10] A. Góźdź and K. Pomorski, Nucl. Phys. A451 (1986) 1.

[11] J. Libert, P. Quentin, Z. Phys. A306 (1982) 315.

[12] T. Seo, Z. Phys. A324 (1986) 43.

[13] J.A. Shannon, W.R. Philips, J.L. Durell, B.J. Varley, W. Urban, C.J. Pearson, I. Ahmed, C.J. Lister, L.R. Morss, K.L. Nash, C.W. Williams, N. Schulz, E. Lubkiewicz, M. Bentaleb, Phys. Lett. B336 (1994) 136.

[14] Q.H. Lu, K. Butler-Moore, S.J. Zhu, J.H. Hamilton, A.V. Ramayya, V.E. Oberacker, W.C. Ma, B.R.S. Babu, J.K. Deng, J. Kormicki, J.D. Cole, R. Aryaeinejad, Y.X. Dardenne, M. Drigert, L.K. Peker, J.O.Rasmussen, M.A. Stoyer, S.Y. Chu, K.E. Gregorich, L.Y. Lee, M.F. Mohar, J.M. Nitschke, N.R. Johnson, F.K. McGowan, G.M. Ter-Akopjan, J.B. Gupta and Y.T. Oganessian, Phys. Rev. C52 (1995) 1348.

[15] J. Skalski, S. Mizutori, W. Nazarewicz, Nucl. Phys. A617 (1997) 282.

[16] J. Eisenberg, W. Greiner in "Nuclear Theory", vol.1, Nuclear Models, NorthHolland Publishing Company (Amsterdam, London 1970).

[17] J. Stachel, P. Hill, N. Kaffrell, H. Emling, H. Grein, E. Grosse, Ch. Michel, H.J. Wollersheim, D. Schwalm, S. Brüssermann, F.R. May, Nucl. Phys. A419 (1984) 589 .

[18] J. Srebrny, D. Cline, C.Y. Wu, R.M. Diamond, D. Habs, H. Huebel, H. Korner, U. Similansky, F.S. Stephens, G.R. Young, C. Bäcktash, Proc. Int. Conf. on Extreme States in Nuclear Physics, Dresden 1980

and

J. Srebrny, T. Czosnyka, D. Cline, C.Y. Wu, A.Bäcklin, L. Hasselgren, C. Falhander, L.B. Svenson, R.M. Diamond, D. Habs, H. Hübel, H. Körner, F.S. Stephens, to be published. 
[19] M.W. Simon, C.Y. Wu, D. Cline, R.W. Gray, M.A. Stoyer, K. Vetter, A.O. Macchiavelli, K.Gregorich, R.M. MacLeod, S. As, Proc. Int. Conf. on Fission and Properties of Neutron-rich Nuclei, Ed. J.H.Hamilton and A.V.Ramayya, World Scientific (1998) p. 270.

[20] NNDC On-line Data Service from the ENSDF database, file revised as of 28th Aug. 1998 - M. Bhat, Evaluated Nuclear Structure Data File (ENSDF), Nuclear Data for Science and Technology, Springer-Verlag, Berlin 1992.

[21] L. E. Svensson, C. Fahlander, L. Hasselgren, A. Bäcklin, L. Wesrerberg, D. Cline, T. Czosnyka, C. Y. Wu, R. M. Diamond, H. Kluge, Nucl. Phys. A584 (1995) 547.

[22] L. E. Svensson "Coulomb excitation of vibrational nuclei", Uppsala Faculty of Science Dissertations, Acta Universitatis Upsaliensis 27, Uppsala 1989 and

L. Hasselgren, D. Cline, Proc. Int. Conf. on Interacting Bose-Fermi Systems in Nuclei, Ed. F. Iachello, Plenum Press (1981) p. 59 (also University of Rochester Nuclear Structure Research Laboratory report UR-NSRL-229).

[23] L. Hasselgren, J. Srebrny, C. Y. Wu, D. Cline, T. Czosnyka, L. E. Svensson, A. Bäcklin, C. Fahlander, L. Westerberg, L. M. Diamond, D. Habs, H. Körner, F. S. Stephens, C. Bäktash and G. R. Young, Proc. 17-th Mikolajki Summer School on Nuclear Physics, Poland 1985 (also University of Rochester Nuclear Structure Research Laboratory report UR-NSRL-338).

[24] T. R. Werner, J. Dobaczewski, M. W. Guidry, W. Nazarewicz. J. A. Sheikh, Nucl. Phys. A578 (1994) 1.

[25] J. Stachel, P. Van Isacker, K. Heyde, Phys. Rev. C25 (1982) 650. 


\section{Figure captions:}

Fig. 1 The pairing vibration mass parameter $\left(B_{\Delta \Delta}\right)$, and potential $\left(V_{\text {pair }}\right)$, and the ground-state function $\left(\Psi_{0}^{N}\right)$ in dependence on the pairing energy gap $\Delta$ for the system of 60 neutrons at the deformation point $\beta=0.2, \gamma=20^{\circ}$. The equilibrium value of the energy gap is $\Delta_{e q} \approx 0.14 \hbar \omega_{0}$, the most probable one is $\Delta_{v i b} \approx 0.09 \hbar \omega_{0}$.

Fig. 2 The collective potential (corrected by the zero-point pairing vibrations) calculated for ${ }^{106-112} \mathrm{Ru}$ isotopes. The energy distance between neighbouring lines is of 1 Mev.

Fig. 3 The experimental [18, 17] and the theoretical (connected by straight lines) excitated levels in ${ }^{104} \mathrm{Ru}$ versus angular momentum $J^{\pi}$. The theoretical values were calculated including the effect of coupling with the pairing vibrations ("new") and without this coupling, i.e. within usual microscopic Bohr model ("old").

Fig. 4 The experimental [18 and the calculated reduced diagonal matrix elements of the quadrupole electric operator in excited states of ${ }^{104} \mathrm{Ru}$. The theoretical points are connected by straight lines.

Fig. 5 Theoretical reduced E2 transition probabilities (white points connected by straight lines) in comparison to the values measured (black points) for the ground-state and $\gamma$ band [17] and for $0_{2}^{+}$band [18] in ${ }^{104} \mathrm{Ru} . J^{\pi}$ means the angular momentum of an initial state.

Fig. 6 The experimental [13, 20] and the theoretical (marked with straight lines) energies of excited levels in ${ }^{106} \mathrm{Ru}$ and ${ }^{108} \mathrm{Ru}$ versus angular momentum $J^{\pi}$.

Fig. 7 The same as in Fig. 5 but for ${ }^{110} \mathrm{Ru}$ and ${ }^{112} \mathrm{Ru}$. Experimental data taken also from [19].

Fig. 8 The experimental [13] and the calculated (marked with straight lines) energies of excited levels in ${ }^{114} \mathrm{Ru}$.

Fig. 9 Theoretical reduced probabilities of E2 transitions between states of ground band (squares) and of $\gamma$ band (triangles) in ${ }^{106-114} \mathrm{Ru}$. Few experimental data known for $2_{1}^{+} \rightarrow 0_{1}^{+}$and $4_{1}^{+} \rightarrow 2_{1}^{+}$transitions [20] are marked with the full squares.

Fig. 10 The predicted diagonal matrix elements of the quadrupole electric operator 
in the states of the ground band (squares) and of $\gamma$ band (triangles) in Ru isotopes.

Fig. 11 The collective potential (including the zero-point pairing vibrations) calculated for ${ }^{106-108} \mathrm{Pd}$. The distance between neighbouring lines is of $1 \mathrm{Mev}$.

Fig. 12 The experimental [21, 22] and the calculated excited levels in ${ }^{106,108} \mathrm{Pd}$ versus angular momentum $J^{\pi}$. The theoretical points are connected by straight lines.

Fig. 13 The experimental [22, 23] and the calculated excited levels in ${ }^{110} \mathrm{Pd}$ versus angular momentum $J^{\pi}$. The theoretical points are connected by straight lines. The calculated levels built on the second $0^{+}$state are interpreted as the members of the $0_{3}^{+}$band (see Section 3).

Fig. 14 The experimental [22, 23] and the calculated reduced probabilities of E2 inband transitions in ${ }^{110} \mathrm{Pd}$. Theoretical points obtained in this work are connected with straight lines. For comparison there are showed results [22] of the IBA-2 calculation (points marked by dotted lines).

Fig. 15 The experimental 22, 23] and the calculated diagonal matrix elements of the quadrupole electric operator in the ground, $\gamma$ and $0_{3}^{+}$bands of ${ }^{110} \mathrm{Pd}$. The theoretical points are connected with straight lines.

Fig. 16 The experimental [21, 22] and the calculated reduced probabilities of E2 inband transitions in ${ }^{106,108} \mathrm{Pd}$. The theoretical points are marked by straight lines.

Fig. 17 The experimental [21, 22] and the calculated diagonal matrix elements of the quadrupole electric operator in the ground and $\gamma$ bands of ${ }^{106,108} \mathrm{Pd}$. The theoretical points are marked by straight lines.

Fig. 18 The experimental [21, 22, 23] and the calculated reduced probabilities of E2 transitions from the $J^{\pi}$ state of the $\gamma$ band in ${ }^{106-110} \mathrm{Pd}$ to the state of the same angular momentum in the corresponding ground band. The theoretical points are marked with straight lines. 
Fig. 1

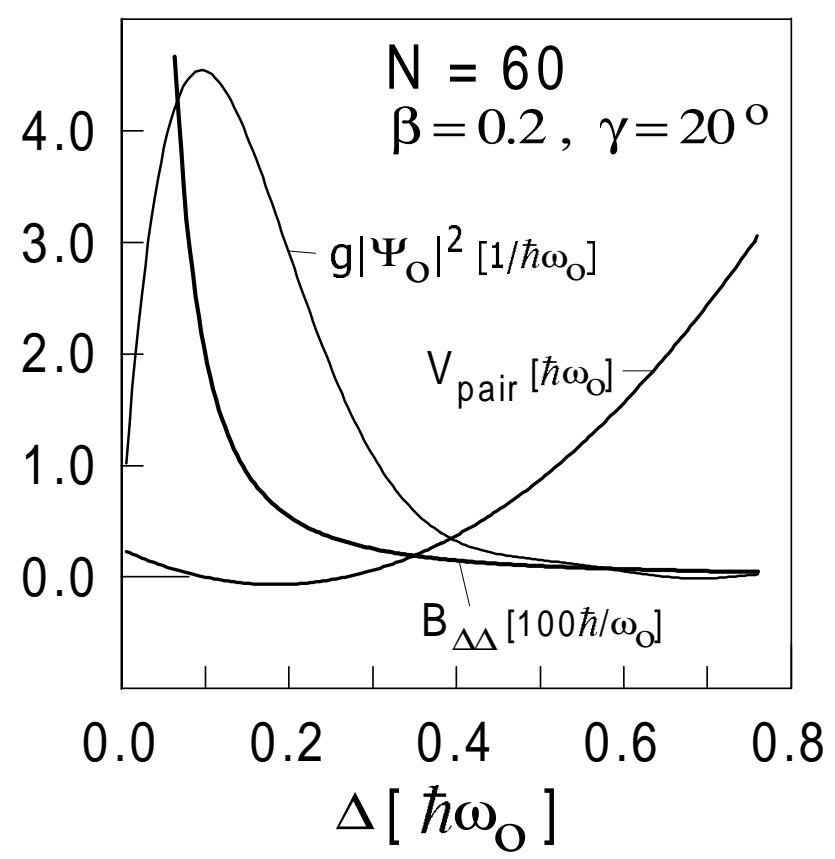



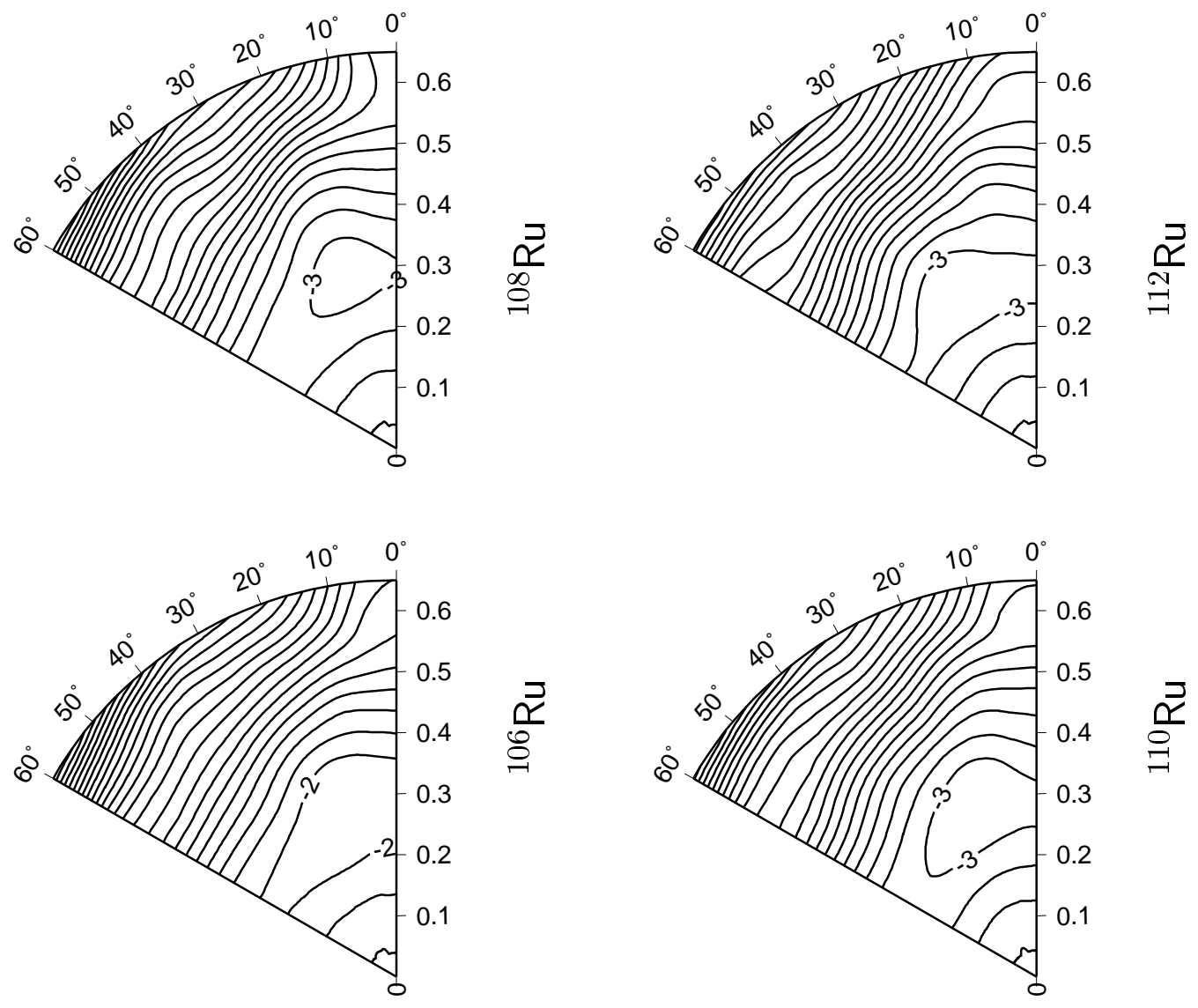


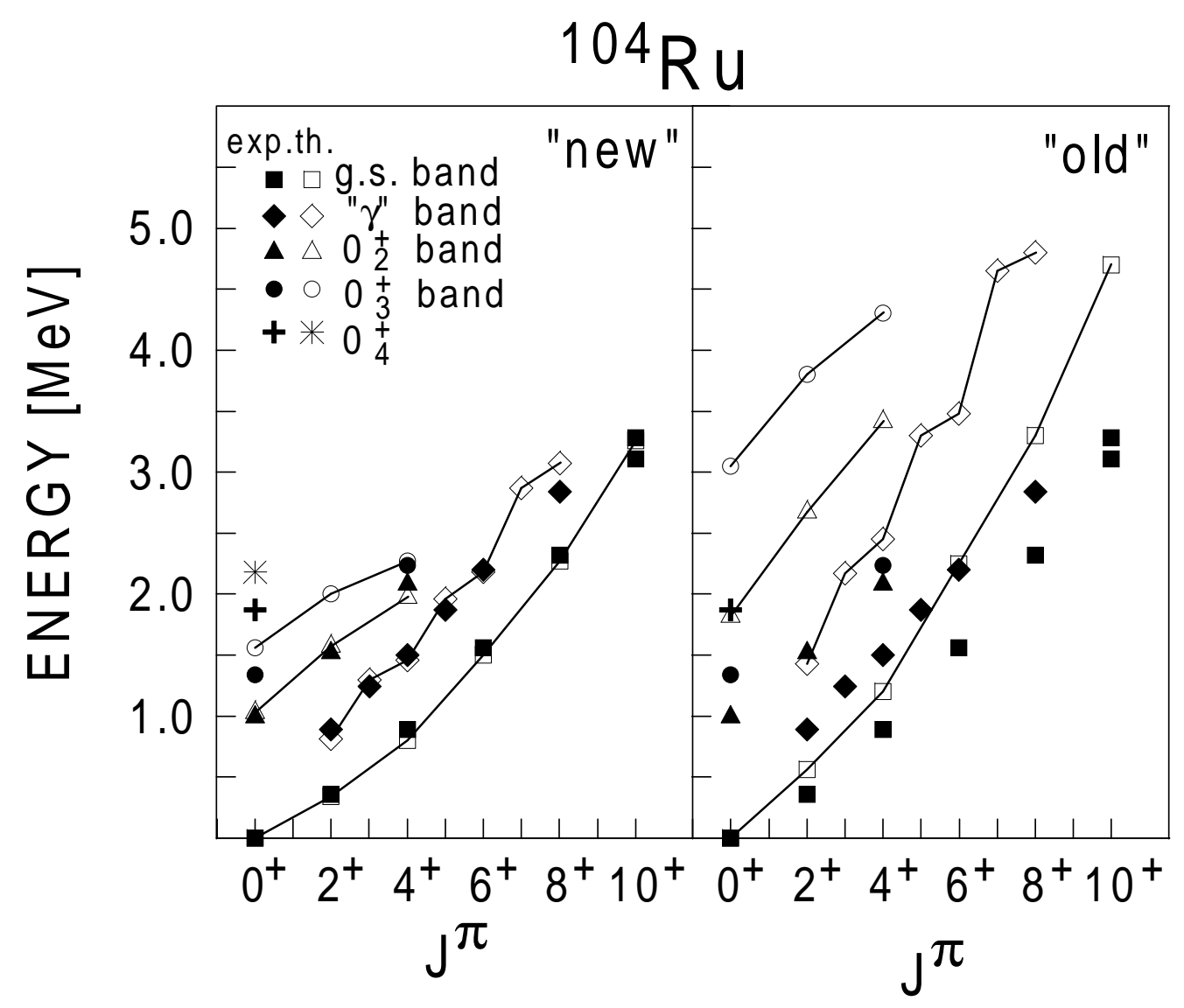


Fig. 4

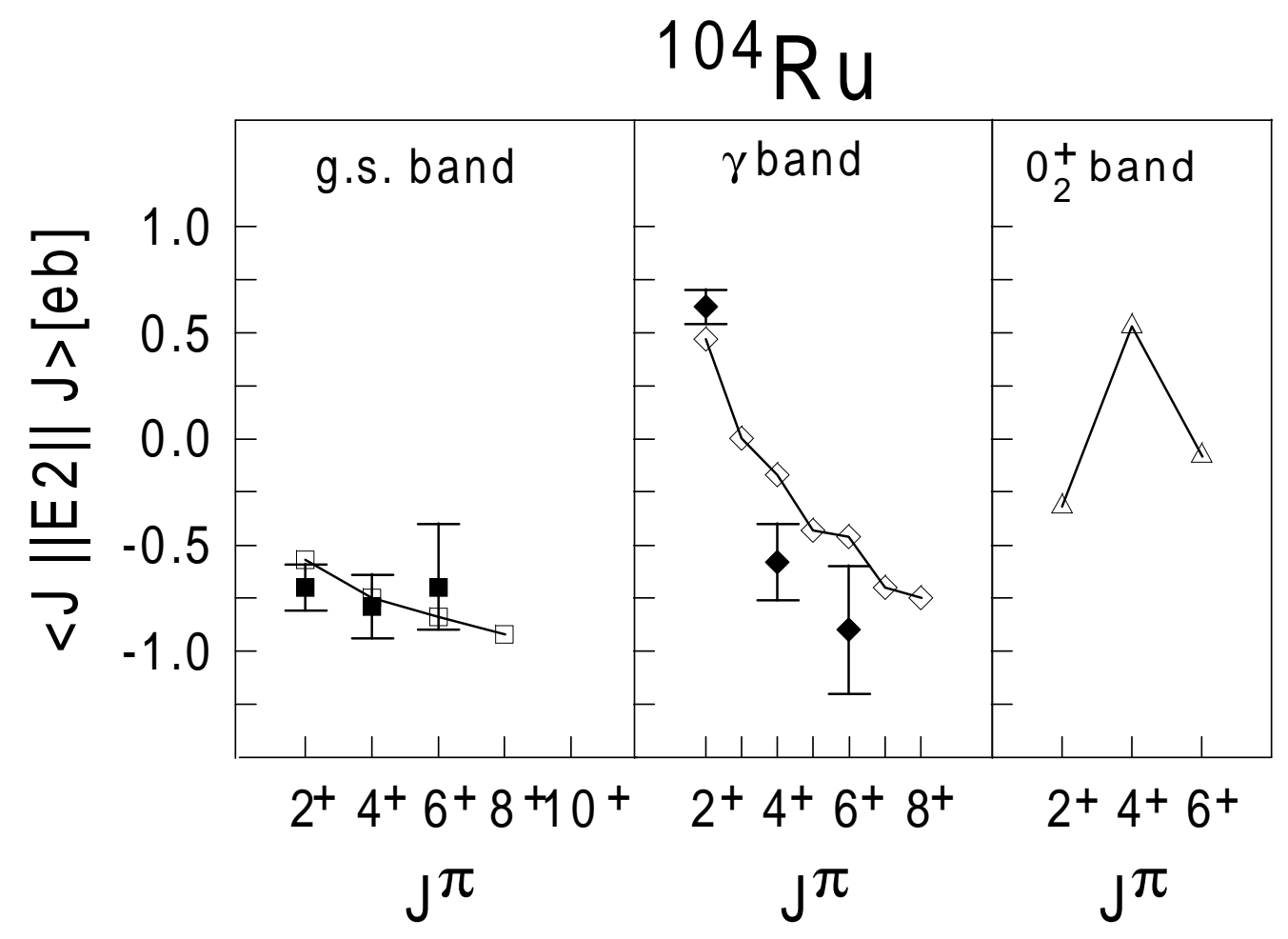




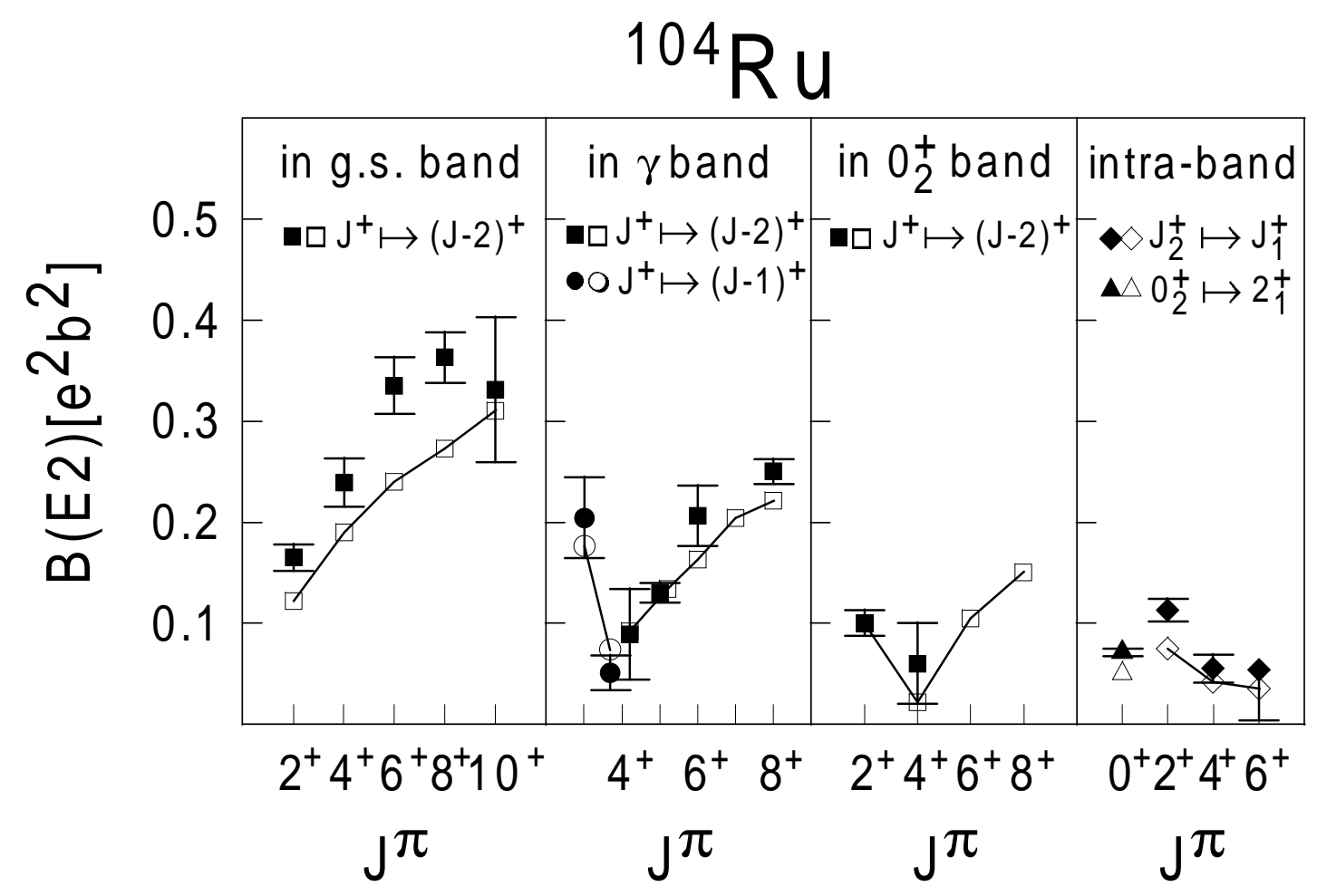


Fig. 6

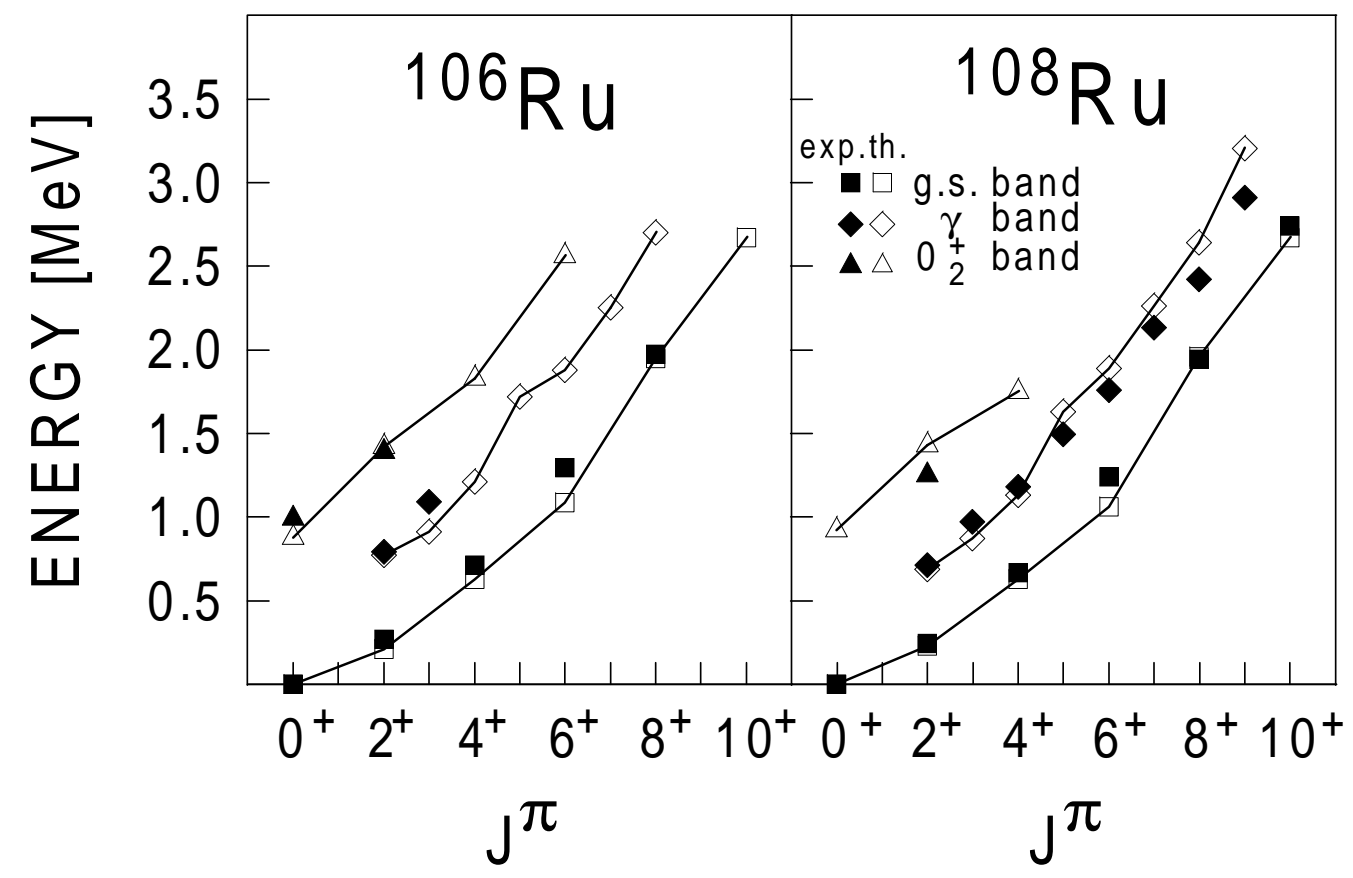


Fig. 7

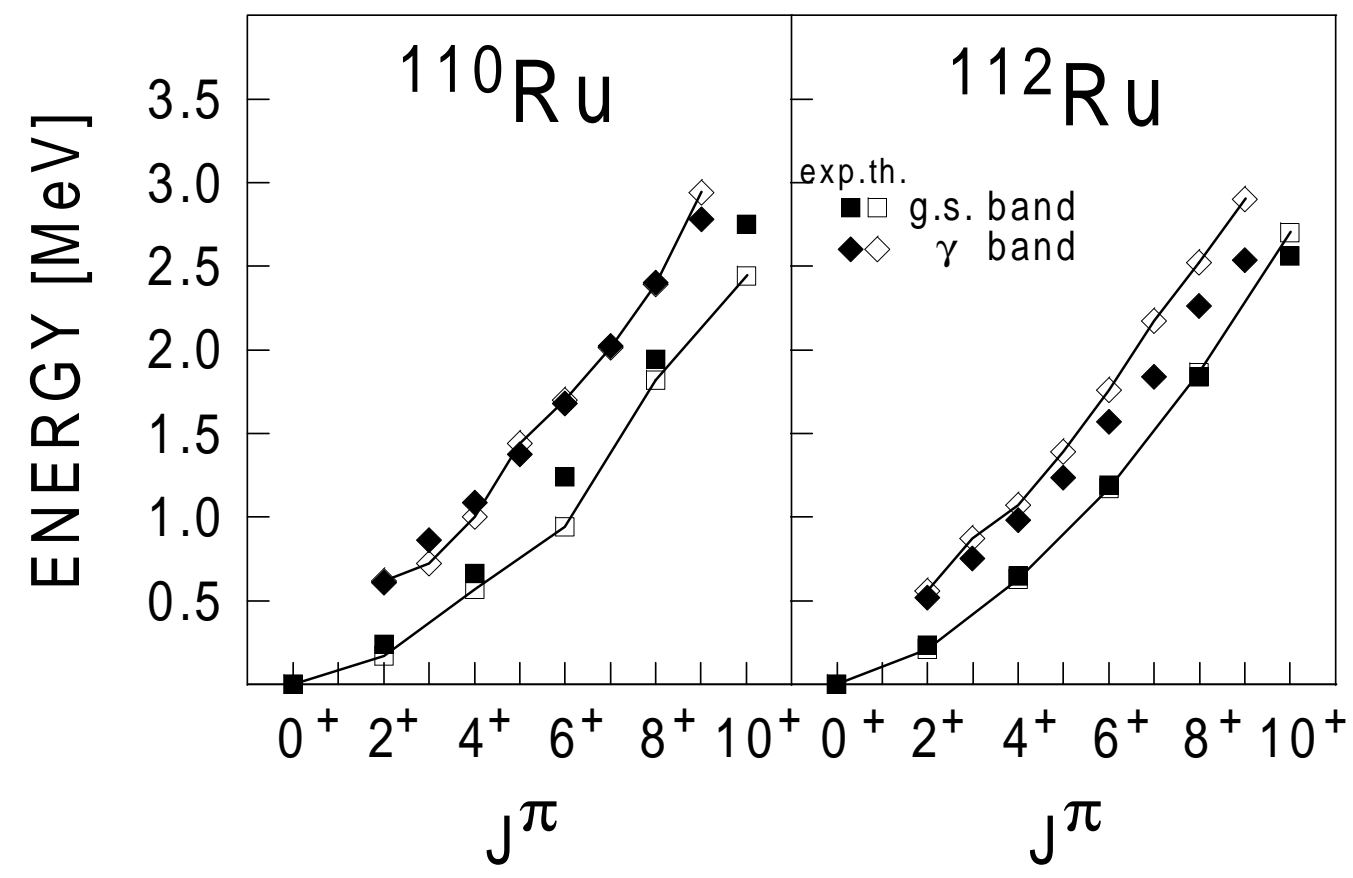


Fig. 8

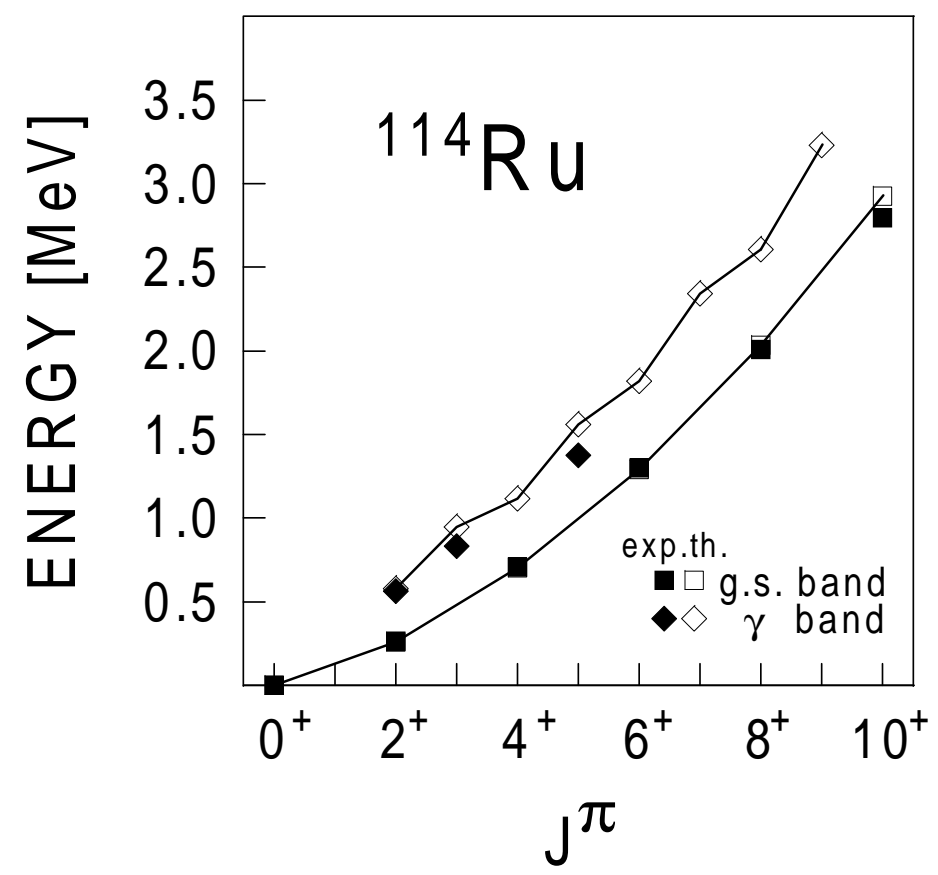


Fig. 9

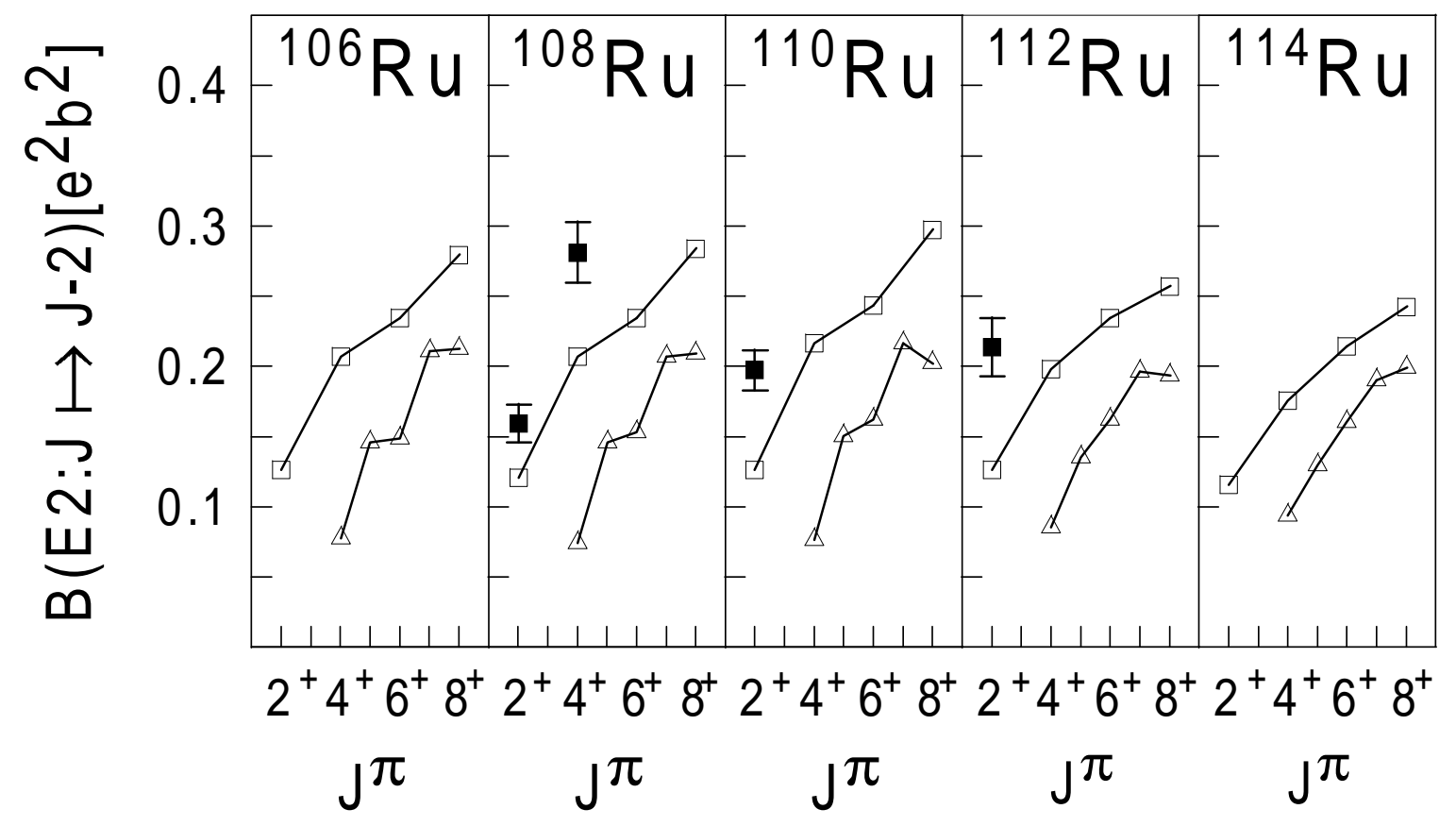


Fig. 10

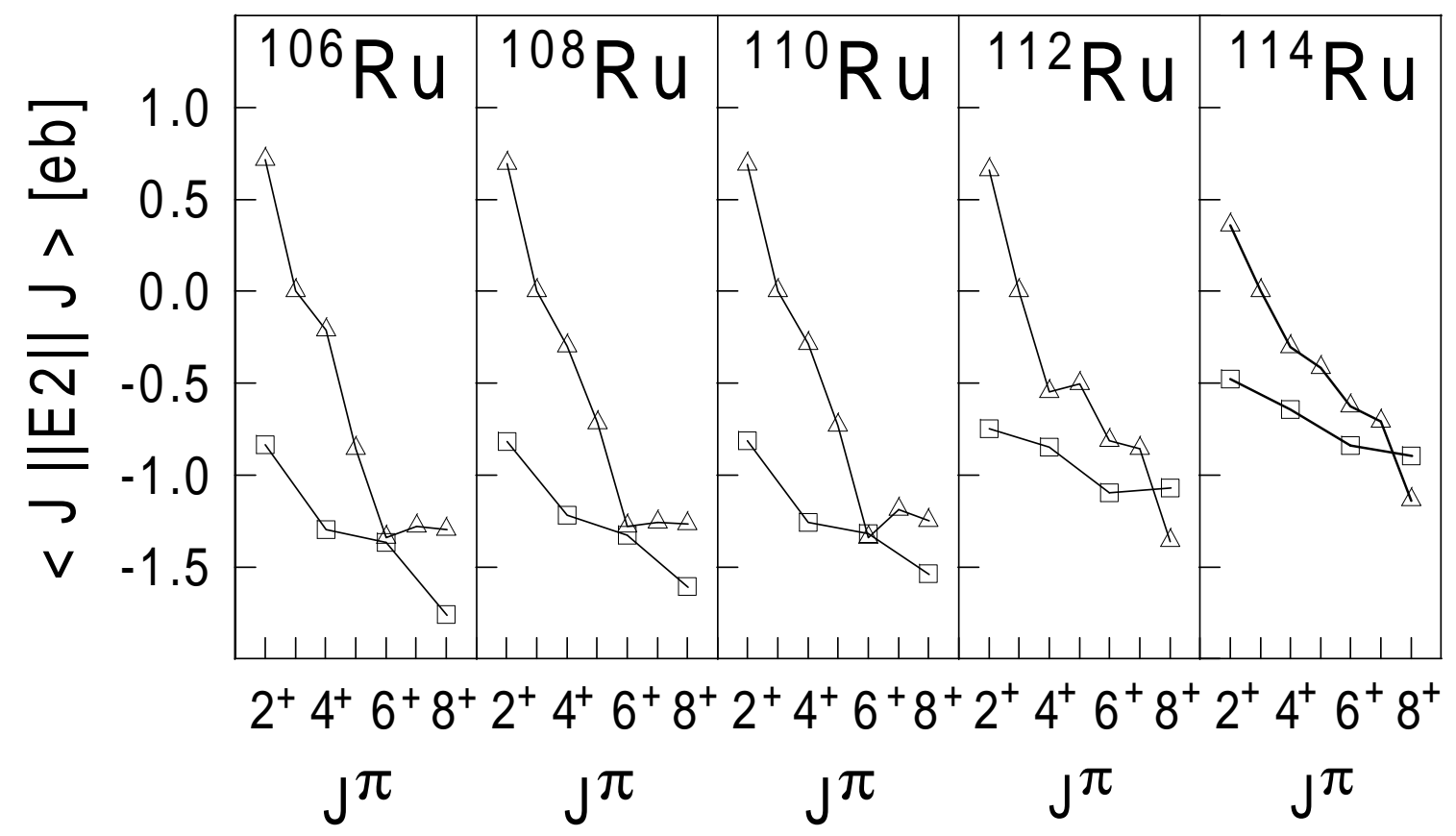




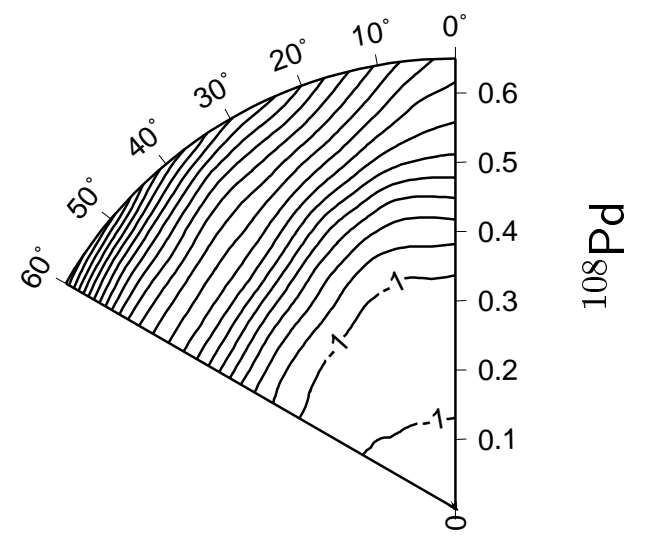

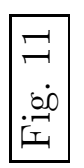

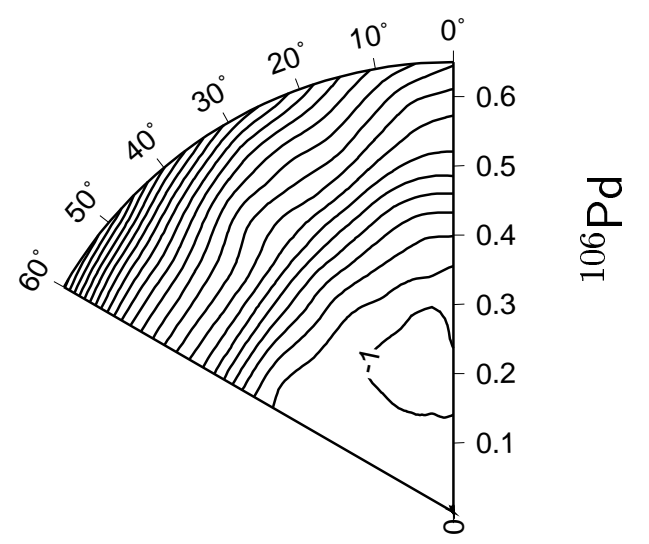




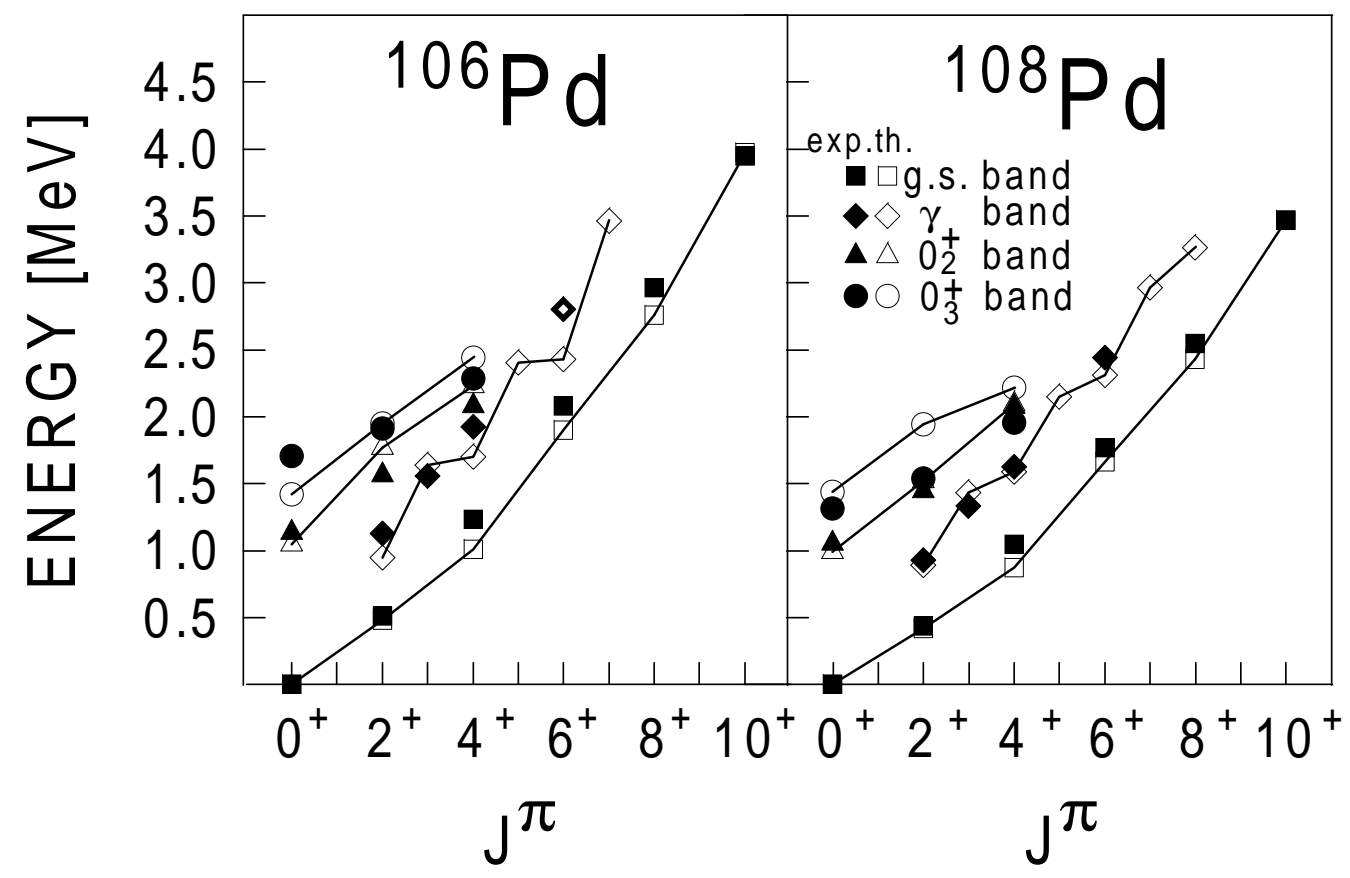


Fig. 13

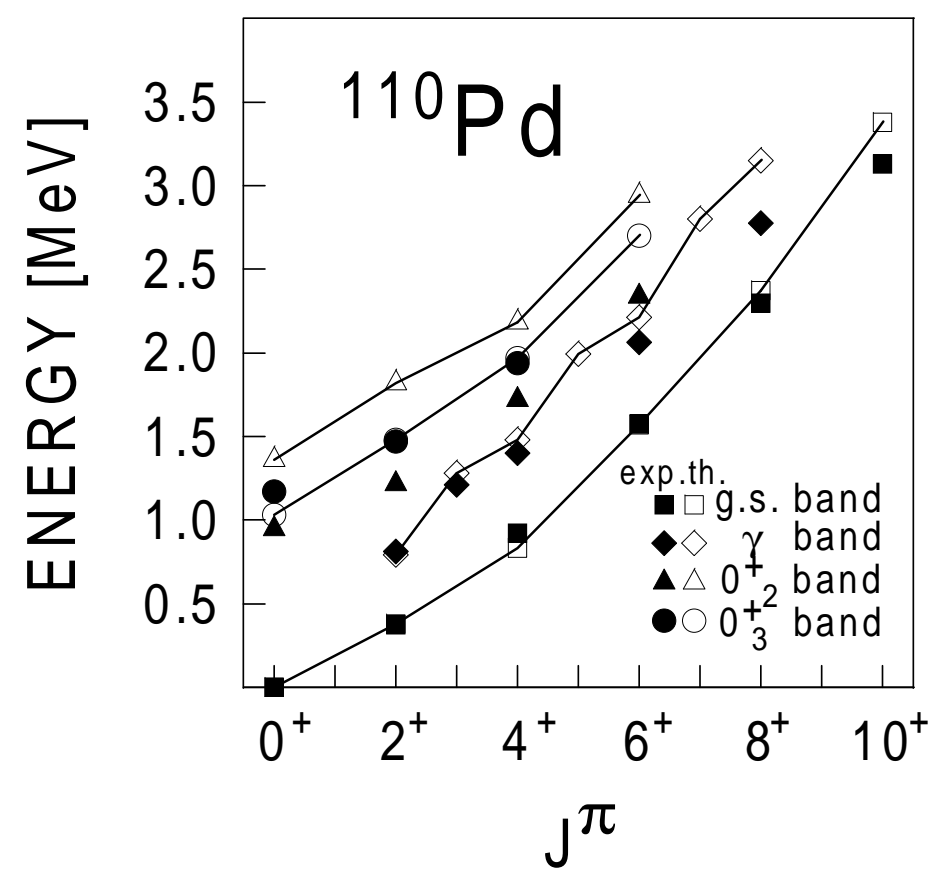


Fig. 14

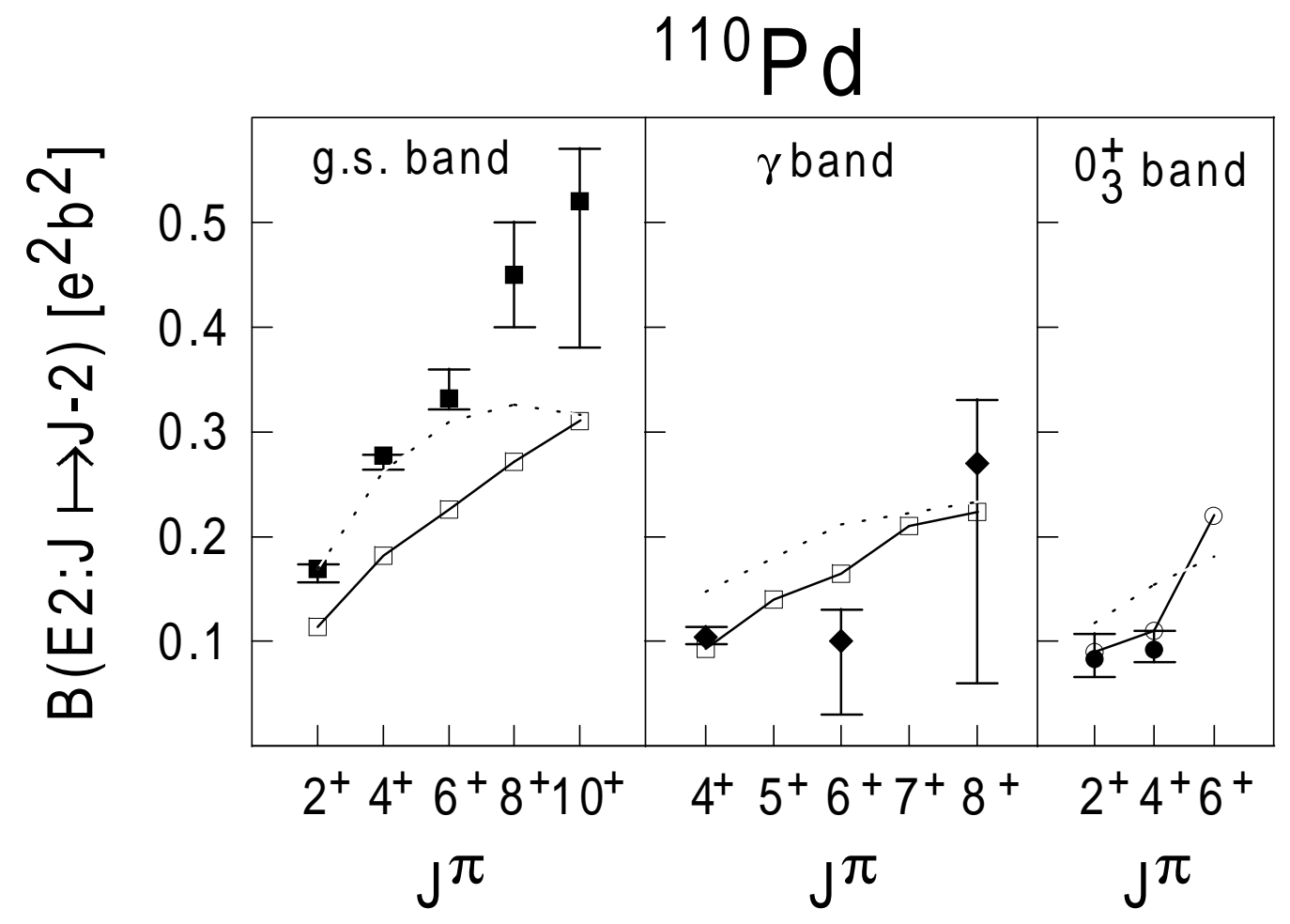


Fig. 15

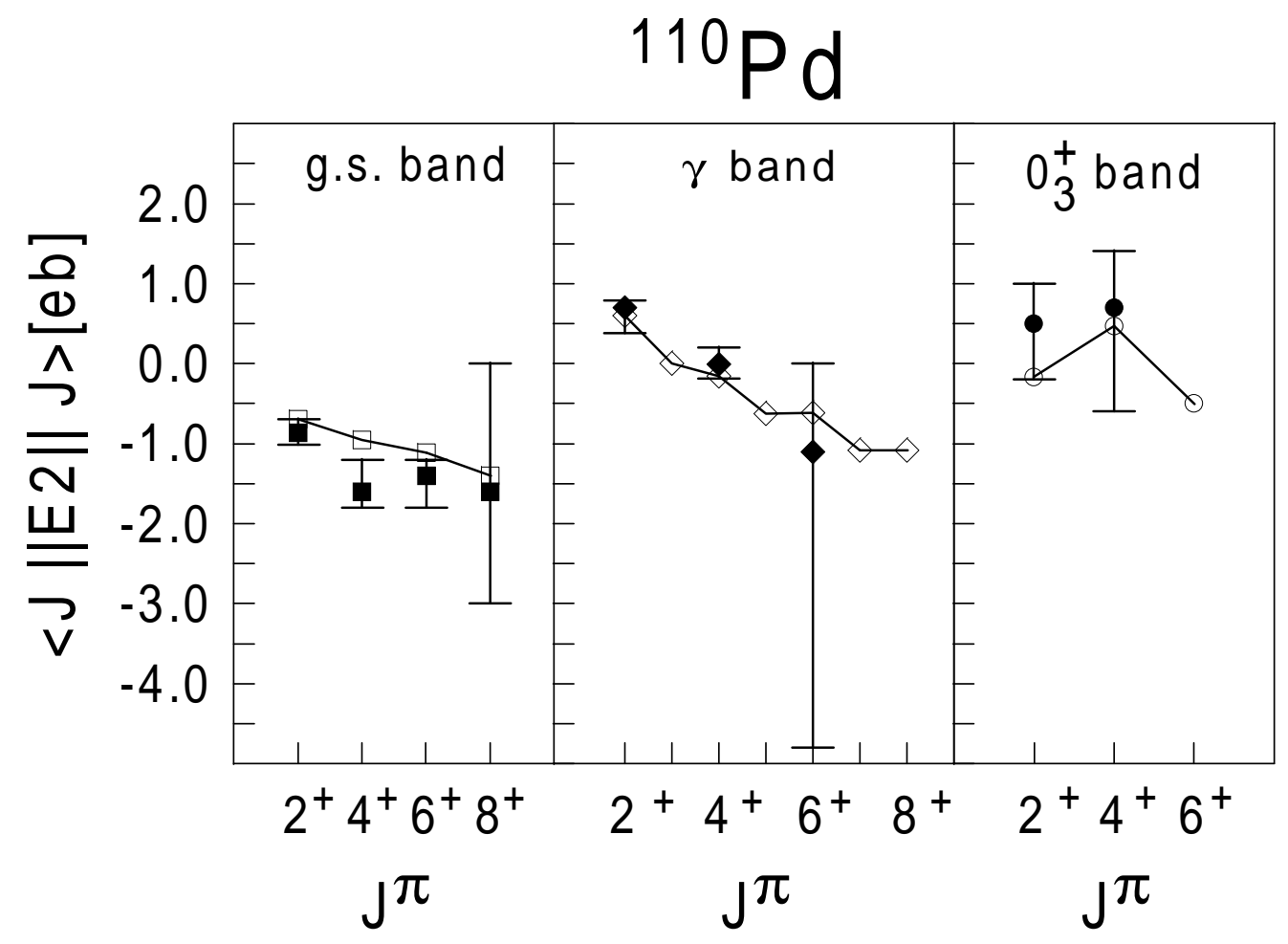




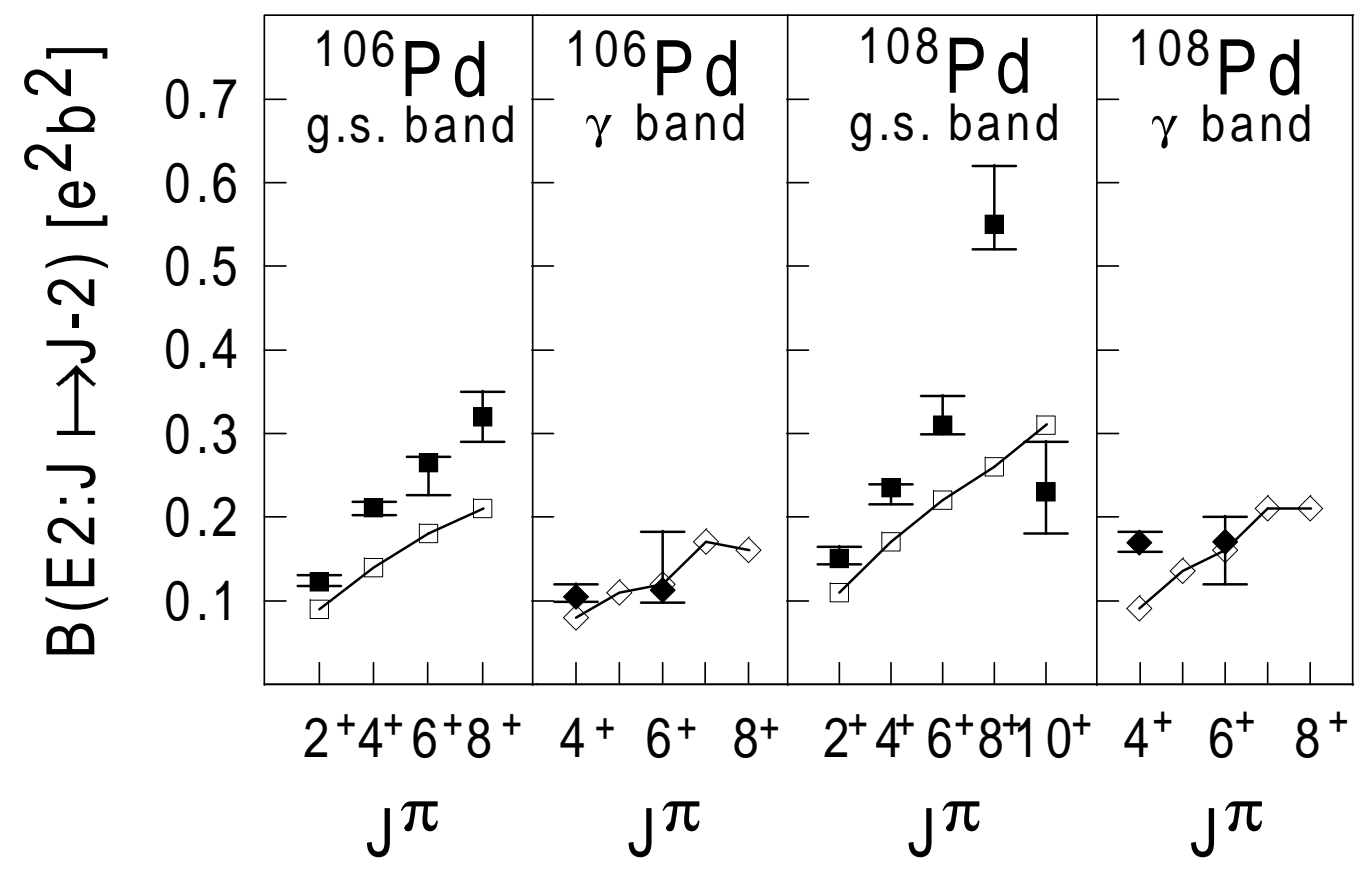


Fig. 17

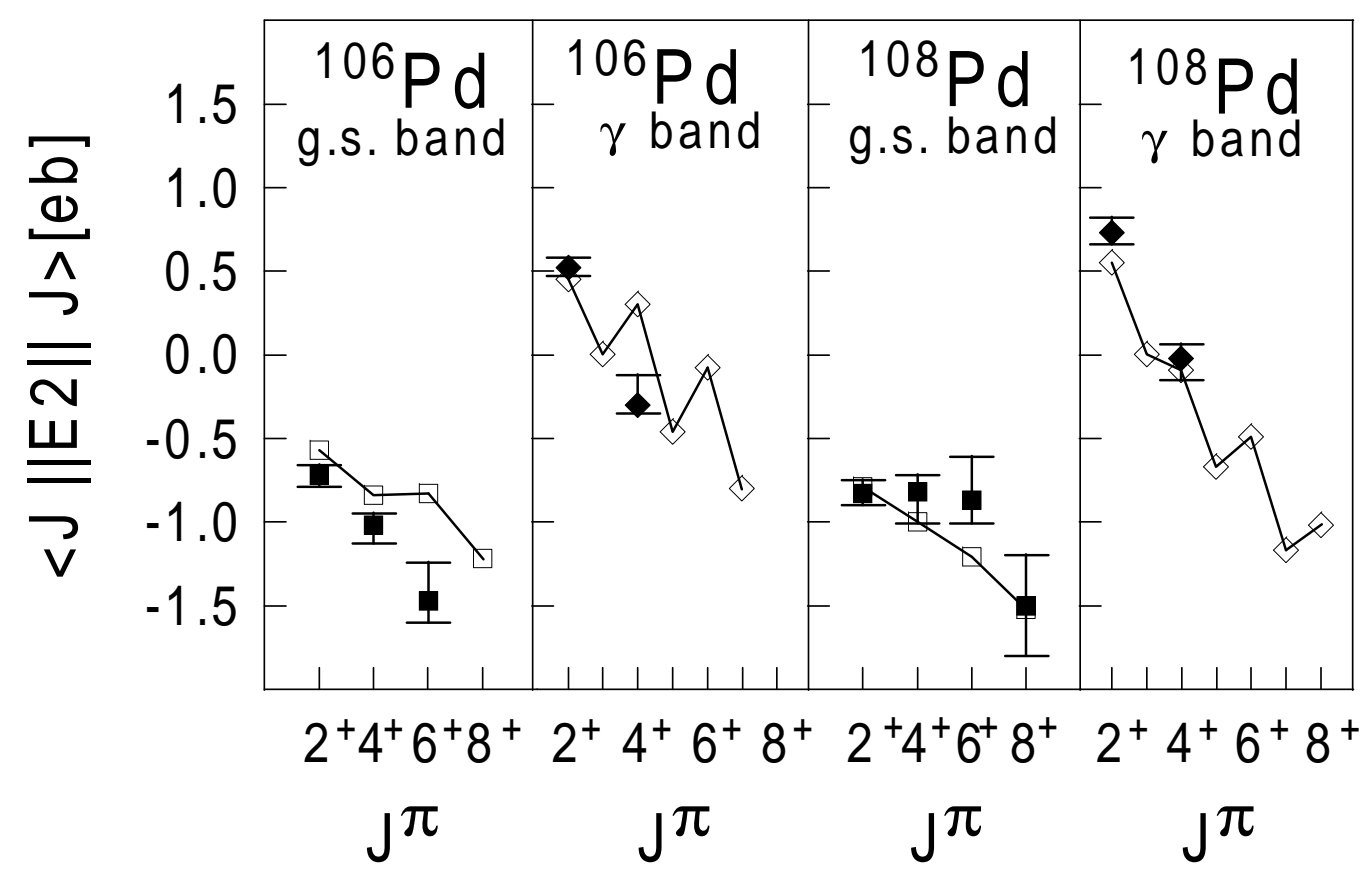


Fig. 18

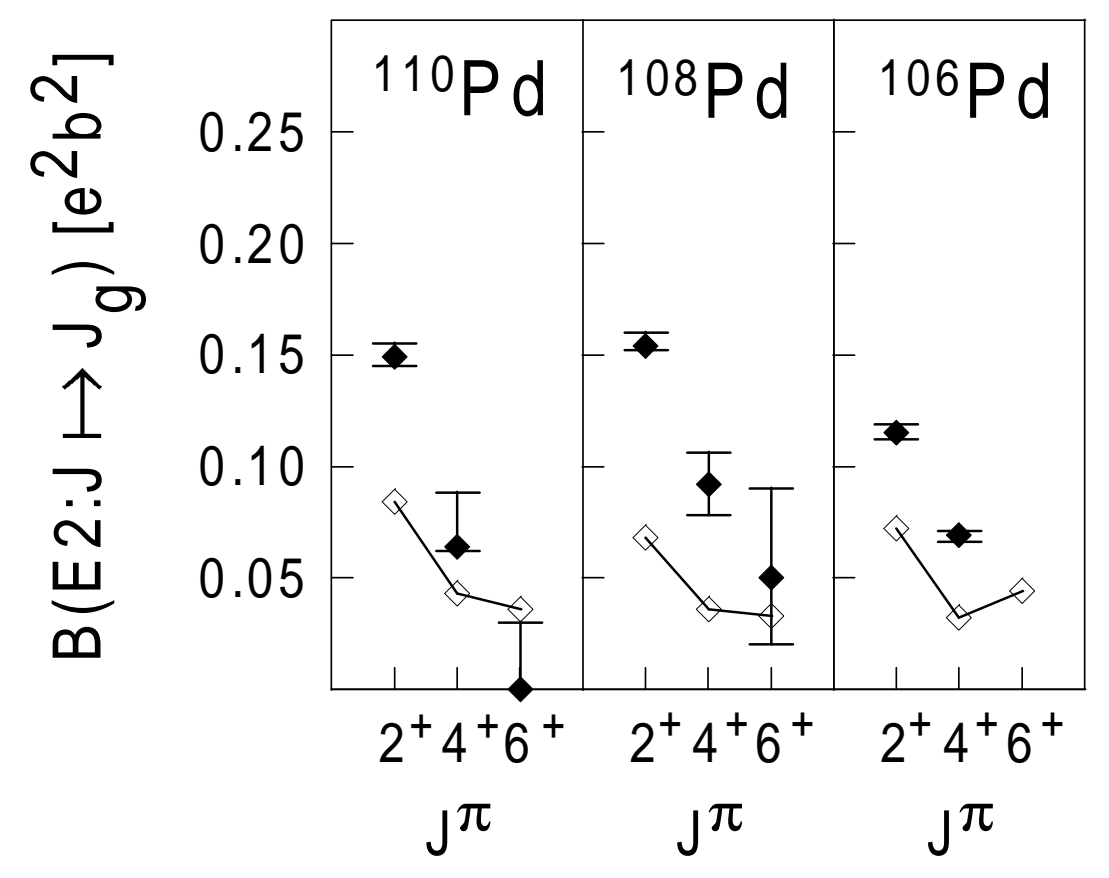

